\title{
COMPARATIVE BANDING PATTERN ANALYSES IN HUMAN CHROMOSOMES INDUCED BY UREA AND TRYPSIN TREATMENTS ${ }^{1)}$
}

\author{
YUKIMASA SHIRAISHI AND TOSIHIDE H. YOSIDA
}

\begin{abstract}
Department of Anatomy, School of Medicine, Kanazawa University, Kanazawa 920, and Department of Cytogenetics, National Institute of Genetics, Misima 411
\end{abstract}

Received September 26, 1972

Studies of banding patterns in human chromosomes have advanced rapidly since the introduction of the quinacrine fluorescence technique (Caspersson et al. 1970, 1971) and several different procedures based upon incubation in warm saline or buffer solutions followed by Giemsa staining (Sumner et al. 1971; Schnedl 1971; Drets and Shaw 1971). These techniques have become available to identify each chromosome pair in the human complement. However, these methods either require special equipment (an ultraviolet microscope) or are time-consuming, and sometimes obscure the banded appearance. A rapid and simple method should therefore be of advantage for the production of banding patterns.

Recently two rather simple kinds of techniques have been developed, which reveal the characteristic banding patterns within chromosomes. The first technique is that developed by Seabright (1971), and Wang and Fedoroff (1972) with trypsin treatment. The second, developed in this laboratory (Shiraishi and Yosida 1971, 1972; Kato and Yosida 1972), has been devised as a result of experiments with urea solution supplemented with saline or phosphate buffer solution. Both techniques were essentially the same as those used for the extraction of protein from the cell. Banding patterns produced by these two techniques appeared to be very similar to each other. The present study was undertaken to compare the banding patterns produced by these two techniques and was also done to confirm the specificity as well as constancy of these patterns.

\section{MATERIALS AND METHODS}

Human peripheral leucocytes from 10 healthy subjects ( 5 females and 5 males) were cultured according to the routine phytohemagglutinin method. Chromosome preparations and the urea banding technique were performed by the same methods as those

1) Contribution No. 933 from the National Institute of Genetics, Japan. Supported by grant-inaid No. 92332 from the Ministry of Education of Japan. 
previously used (Shiraishi and Yosida 1972).

The technical procedure for trypsin banding was performed according to Seabright (1971) but modified slightly. Within 24 hours after air drying, chromosome preparations were treated for $5-10$ seconds with cold trypsin-versene $(0.05 \%$ trypsin solution in $0.02 \%$ EDTA solution) kept in refrigerator $\left(0-4^{\circ} \mathrm{C}\right)$. Then, the slides were rinsed thoroughly with tap water and stained with Giemsa diluted about 50 times with Sörensen's buffer (pH 6.8) for 5-10 min. The trypsin treatment needed some cautions in handling. When freshly prepared slides were immediately immersed in the treatment solution, most of bands appeared to be obscure or took a fuzzy appearance, which was no longer informative. This could be avoided to a certain degree by drying the slides for many hours. Best results were obtained when slides were kept drying overnight (for about 12 hours) at room temperature before use. Natural drying is desirable for these methods.

\section{RESULTS}

Banding patterns of normal female and male karyotypes produced by these treatments are presented in Figures 1 to 3 . Those produced by the urea and trypsin treatments are shown in "a" and " $b$ " in these figures, respectively. As seen in the figures, each chromosome pair showed a specific pattern of bands within chromosomes and close similarity in homologues, and the patterns produced by the two methods seemed to be essentially the same, though the pattern produced by the urea technique is slightly clearer than that produced by trypsin. A diagram depicted in the last column of Figs. 1-3 was determined based on more than 40 suitable metaphase cells. Banding patterns produced by the present two methods were in general good agreement with those obtained in our previous work (Shiraishi and Yosida 1972). Chromosome pairs 4, 5, $9,11,12,19,20,21$ and 22 in the previous description were designated in the present study as pairs No. $5,4,11,12,9,20,19,22$ and 21 , respectively, referring to the banding patterns published by Caspersson et al. (1970, 1971), Seabright (1971) and Paris Conference (1971). In the present study, diagrams of banding patterns in pairs 5 and 13 and the short arm of the $\mathrm{X}$ chromosome were slightly revised as follows. Chromosome 5 shows in its long arm five dark bands and two dark bands in the short arm. The three characteristic dark bands in the middle of the long arm are helpful for identification. Close to the distal part of both arms and the centromere of the short arm unstained regions are visible. Chromosomes 4 and 5 display a close morphological similarity, but they are distinguishable by the banding patterns (Fig. 1). Chromosome 13 has three or four dark bands in the long arm. The distal part of the long arm is sometimes unstained. The X-chromosome exhibits three dark bands in the long arm and one dark band in the middle of the short arm. The distal part of the short arm is unstained. No difference has been observed between the two X-chromosomes in the female. 
COMPARATIVE BANDING PATTERN ANALYSES

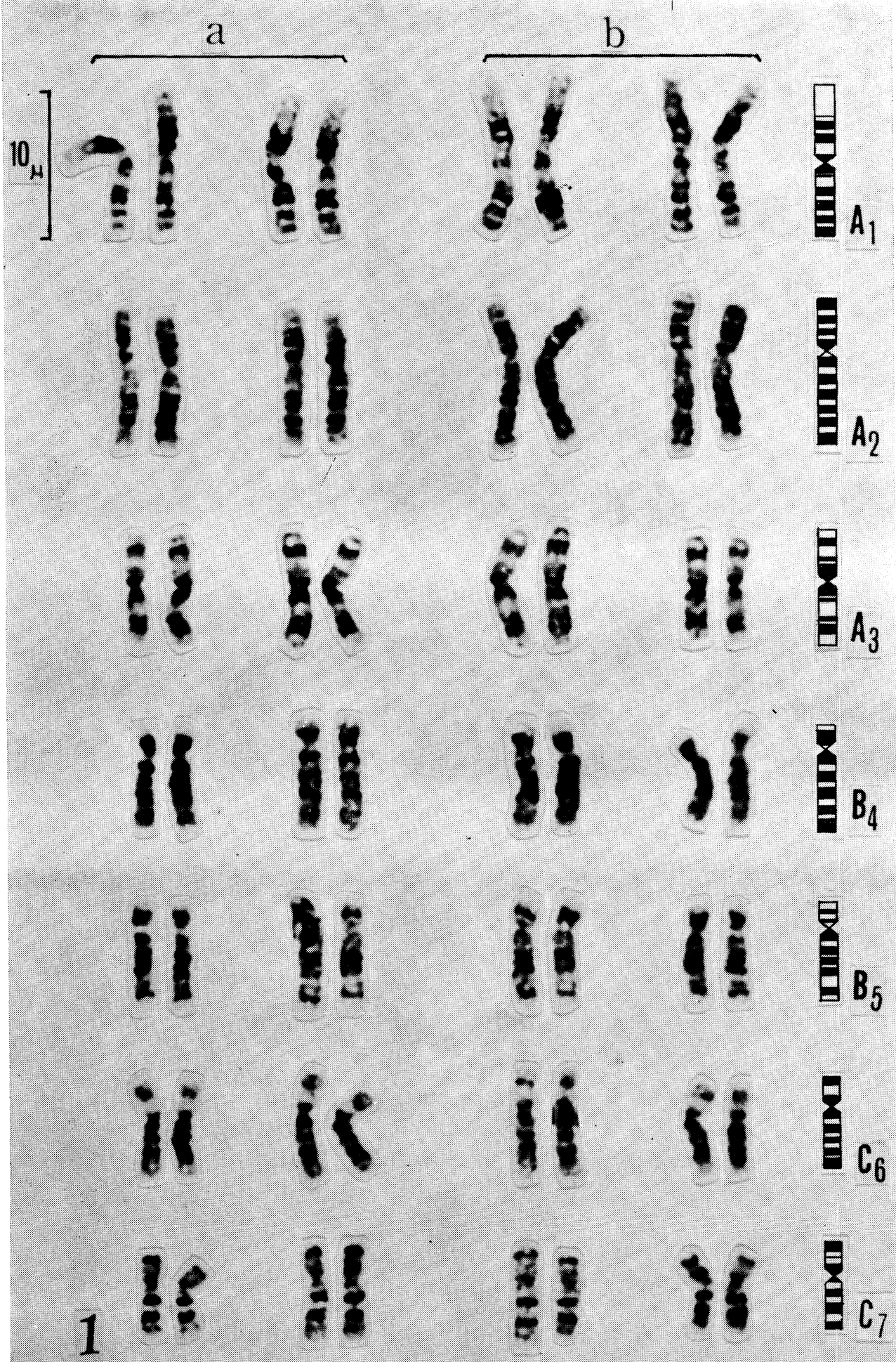



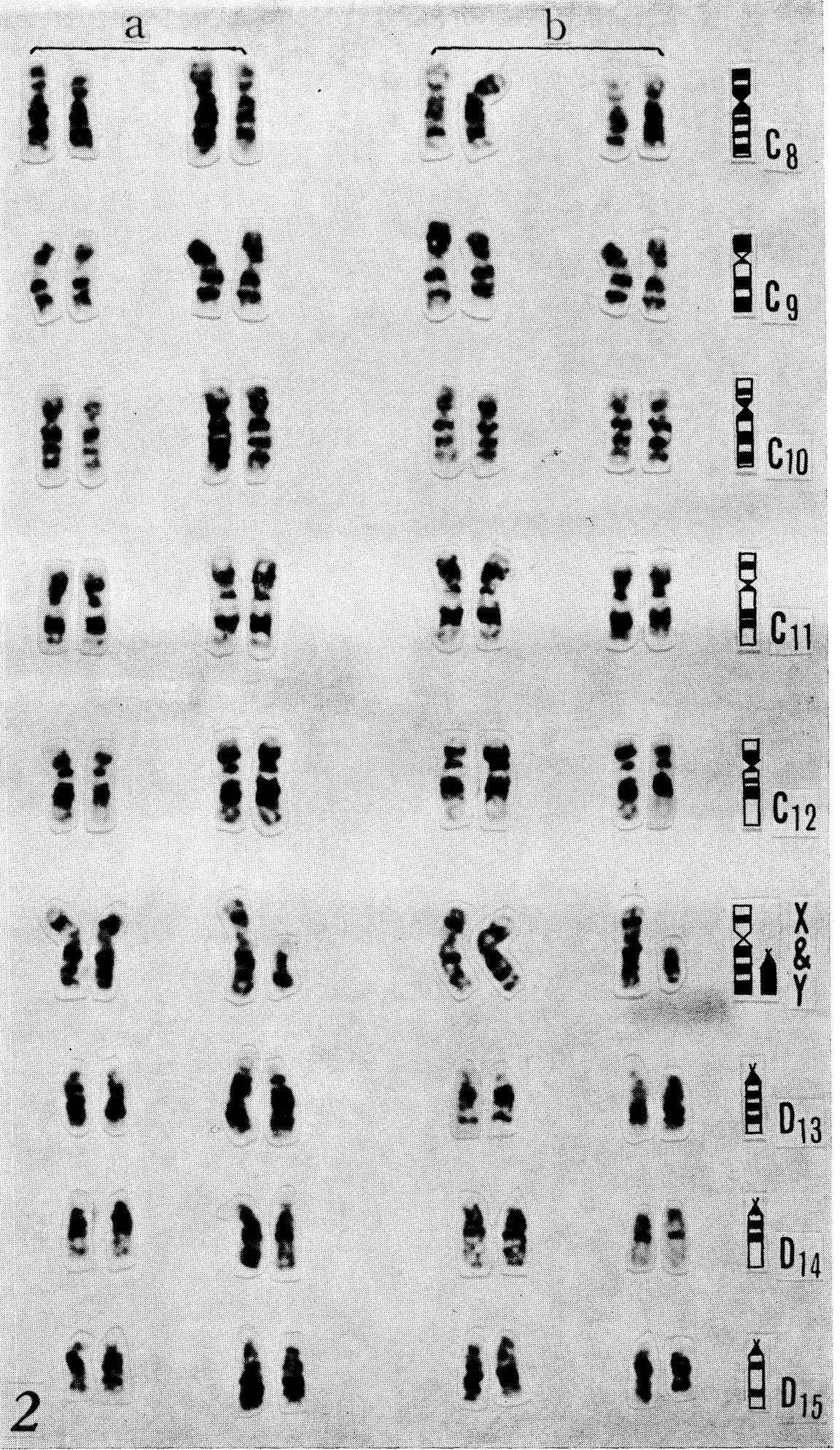


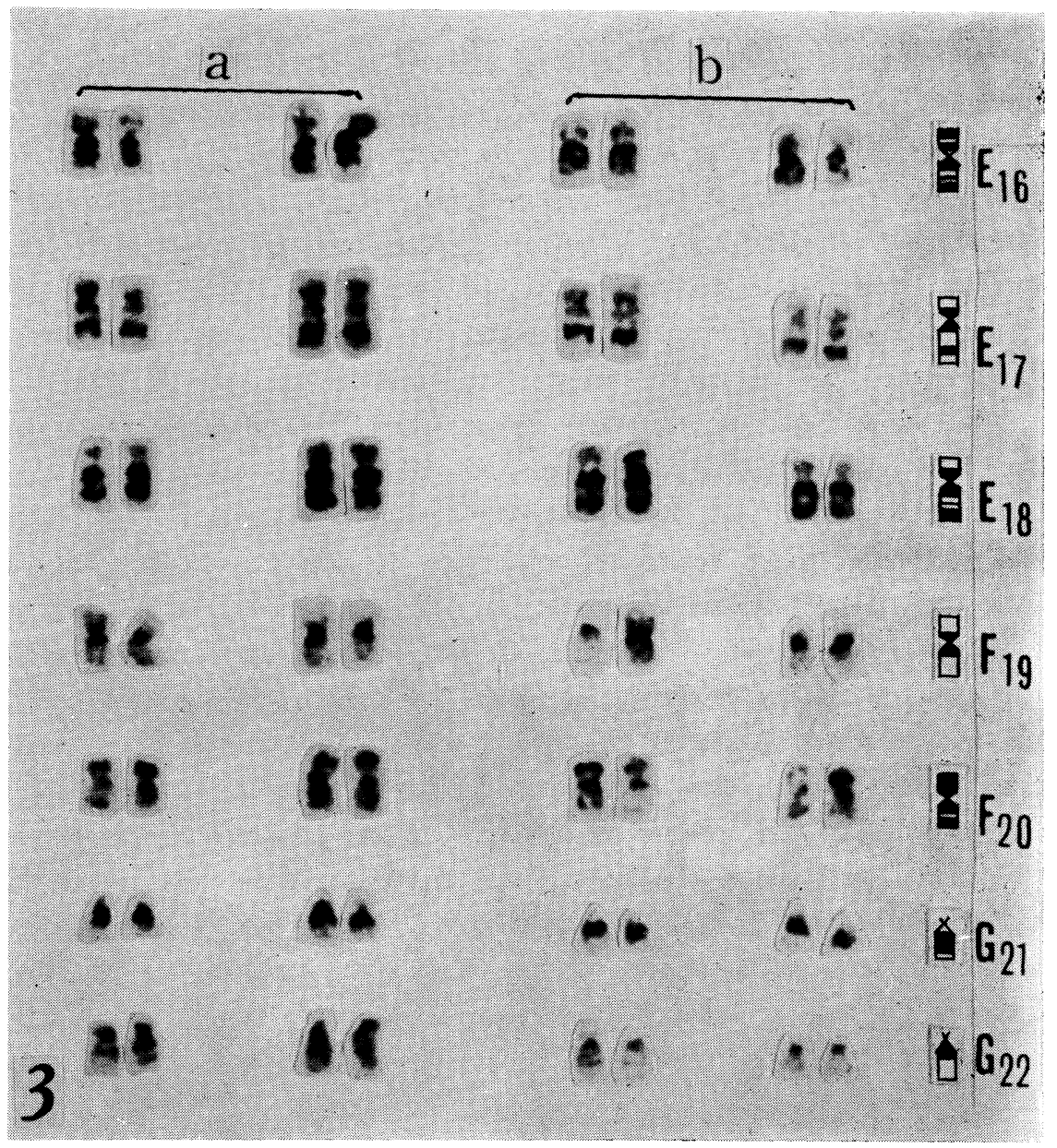

Figs. 1-3. Four normal karyotypes of females and males after treatment with two techniques and diagram of most reliable patterns for each chromosome pair.
a. Urea-treatment
b. Trypsin-treatment

\section{DISCUSSION}

There was no difference in banding pattern between the urea and trypsin treatment techniques. The patterns produced by these two methods are also similar to those shown by quinacrine fluorescence (Caspersson et al. 1970, 1971) and other techniques by Giemsa staining (Sumner et al. 1971; Schnedl 1971; Drets and Shaw 1971). In contrast to the fluorescence technique, dark stained bands can also be detected by the present techniques at the centromere regions of most chromosomes. A striking peculiarity is found in the secondary constriction adjacent to the centromere of the long arm of chromosomes 1 and 16 . The regions, very poorly stained with quinacrine, are darkly stained by the present techniques. 
The most important observation in the present study was that the procedures described induced the characteristic banding pattern in all individual human chromosomes, and these patterns enabled the identification of all chromosome pairs. Although the chromosomes in the groups $\mathrm{A}$ and $\mathrm{E}$ are ascertained from arm ratio and chromosome length, the chromosome pairs within groups B, C, D, F and G display rather close morphological similarities. It is therefore necessary and important to standardize the system of chromosome numbering in groups B, C, D, F and G based upon the characteristic banding patterns in each chromosome pair. Consequently the banding patterns are very helpful for accurate identification and designation of individual chromosomes and detection of specific chromosome segments involved in rearrangements such as translocations and inversions (Paris Conf.).

The procedures used in the present study are essentially the same as those employed for protein extraction from cells. Although we do not know the exact molecular mechanisms, the banding patterns revealed by Giemsa staining technique might be related to the physical state of constituent DNA and its association with proteins, rather than to the denatuation and renaturation of DNA. Recently Wang and Fedoroff (1972) suggested that removal of protein component of the nucleoprotein complexes making up the chromosomes might allow Giemsa stain to react directly with the nucleic acid component. According to Kato and Moriwaki (1972) the solubilization or extraction of some chromosomal proteins, probably of acid nature, would be the primary cause of the appearance of banded structure of metaphase chromosomes. However, the relation of the banding patterns to functional aspects of specific chromosomes requires further study.

\section{SUMMARY}

The present study was undertaken to compare the banding patterns of human chromosomes produced by trypsin and urea treatment techniques. Banding patterns produced by these two techniques appeared to be essentially the same and specific in each pair of homologues. These patterns also enabled the recognition of specific segments of all individual human chromosomes. From the banding pattern produced by the two techniques a diagram of the pattern was proposed by a slight modification from our previous presentation (Shiraishi and Yosida 1972).

\section{ACKNOWLEDGMENTS}

The authors are indebted to Associate Prof. Dr. T. Matsuda for his helpful advice with special interest and reading the manuscript. 


\section{LITERATURE CITED}

Caspersson, T., C. Johansson, and E. J. Modest, 1970 Identification of human chromosomes by DNA-binding fluorescent agents. Chromosoma (Berl.) 30: 215-227.

Caspersson, T., G. Lomakka, and L. Zech, 1971 The 24 fluorescence patterns of the human metaphase chromosomes-distinguishing characters and variability. Hereditas (Lund) 67: 81-102.

Drets, M. E., and M. W. Shaw, 1971 Specific banding patterns of human chromosomes. Proc. Nat. Acad. Sci. (Wash.) 68: 2073-2077.

Kato, H., and T. H. Yosida, 1972 Banding patterns of Chinese hamster chromosomes revealed by new techniques. Chromosoma (Berl.) 36: 272-280.

Kato, H., and K. Moriwaki, 1972 Factors involved in the production of banded structures in mammalian chromosomes. Chromosoma (Berl.) 84: 105-120.

Paris Conference, 1971 IVth International Conference on Standardization in Human Cytogenetics.

Schnedl, W., 1971 Analysis of human karyotype using a reassociation technique. Chromosoma (Ber1.) 34: 448-454.

Seabright, M., 1971 A rapid banding technique for human chromosomes. Lancet, ii: 971-972.

Shiraishi, Y., and T. H. Yosida, 1971 Differential staining of human chromosomes by treatment with urea. Proc. Japan Acad. 47: 729-731.

Shiraishi, Y., and T. H. Yosida, 1972 Banding pattern analysis of human chromosomes by use of a urea treatment technique. Chromosoma (Berl.) 37: 75-83.

Sumner, A. T., H. J. Evans, and R. A. Buckland, 1971 New technique for distinguishing between human chromosomes. Nature New Biol. 232: 31-32.

Wang, H. C., and S. Fedoroff, 1972 Banding pattern in human chromosomes treated with trypsin. Nature New Biol. 235: 52-53. 